$\mathrm{He}$ has succeeded in calculating the minimum energy needed by the incident particle to produce this effect, and finds that the order of magnitude is in quite good agreement with the experimental observations of cosmic ray energies.

It is hoped that this new conception of the interaction between elementary particles will suggest methods of solving the difficulties in the theory of quantum electrodynamics, which arise from the incomplete knowledge of the physical laws governing the behaviour of particles of high energy.

V. Weisskopf discussed the Dirac theory of positrons, and showed that its formulation can be simplified, and some of its paradoxes removed. P. Jordan discussed the theoretical possibility of conceiving a light quantum as a pair of neutrinos. M. Born gave an account of his theory of quantum electrodynamics. Kalckar and Bohr gave a detailed mathematical account of their theory of nuclear disintegration. O. R. Frisch and G. Placzek gave an account of the structures of energy levels in heavy nuclei, and the possibility of investigating it by means of the absorption of slow neutrons. Rosenfeld and Bohr discussed the problem of the measurement of charges, and the importance of field and charge fluctuations for the definition of theoretical concepts.

The course of the discussions was, as always, directed by the masterly fundamental criticisms of Bohr. On these occasions, when so many able theorists are gathered together, it is clear that Bohr's physical insight is the power which contributes most to the advance of theoretical atomic physics, and creates the conditions which fertilize the mathematical abilities of theoretical workers elsewhere.

The most interesting and important paper on experimental physies was, perhaps, Jacobsen's account of his repetition of the Shankland experiment (see p. 24 of this issue). Shankland failed to observe coincidences between recoil electrons and scattered quanta when observing with counters at the angles expected according to the Compton theory of the photon scattering process. He used $\gamma$-rays from radium, which cover a large range of frequencies. Jacobsen, and Bothe and Maier-Leibnitz have now repeated the experiment with the almost non-chromatic $\gamma$-rays from thorium $\mathrm{C}^{\prime}$. They have taken great care in defining the angles used, and have found the expected number of coincidences. These results are confirmed by some less well-defined experiments by Fermi's collaborators. Thus the suggested failure of the conservation of energy in the Compton effect, so eagerly seized upon by Dirac, in order to get rid of some of the difficulties found in the formulation of a satisfactory relativistic quantum dynamics, is not confirmed. Bohr, Pauli, Heisenberg and other theoretical workers expressed satisfaction at this result, and hoped that further discoveries would mitigate the difficulties on which Dirac comments (see p. 24).

Heitler reported that Anderson has withdrawn his claim that the energy lost by fast electrons in the cosmic rays is much less than expected on general theoretical grounds.

Miss Meitner gave an account of some work on the radioactive effects produced in uranium by the action of slow neutrons. She showed that it is very probable that elements of atomic number 93 and 94 are both formed in the process. Dr. M. L. Oliphant described some recent work carried out in Cambridge, while Goldhaber showed that the mean free path in paraffin of the neutrons produced by the photo-electric disintegration of deuterium does not have the value predicted by the accepted theoretical picture of the interactions between neutrons and protons.

Uhlenbeck described how the modification of the Fermi theory of $\beta$-decay, proposed by Uhlenbeck and Konopinski, is in good accord with experiments on the energy distribution of $\beta$-particles from both electron and positron emitters of low atomic number. Richardson gave similar evidence in the case of $\beta$-emitters of high atomic number.

The general impression left by the conference was that progress in experimental research on the atomic nucleus is very rapid, but that the advance in the theoretical description of the new results is much slower. This is due largely to inadequate theories of the interaction forces between particles of high energy separated by small distances, which are of fundamental importance in the region of the nucleus.

\title{
Recent Advances in Wool Research
}

CEVERAL papers read at the Annual Conference of the Textile Institute held in London on June 3-5 dealt with wool and its characteristies.

Dr. A. B. Wildman, biologist of the Wool Industries Research Association, discussed estimations in the fleece of important wool characteristics, such as fineness variability, length, fleece density and kemp proportion. Emphasis was laid on the necessity for devising speedy and accurate methods of fleece analysis, in order that the relative merits of fleeces from breeders' flocks and from experimental sheep could be accurately measured. A historical résumé of earlier work was given, indicating that conflicting results were often due to lack of representative sampling methods. The author gave an account of his methods, which are subject to statistical control, in commencing analyses of thoroughly sampled fleeces. These investigations are intended to show the kind of variations occurring in fleeces of different types of sheep, and represent an essential prerequisite in the evolution of suitable methods for quality determination.

The quality of a fleece may be modified by exposure to light, air and water. This is attributed by Dr. J. B. Speakman, of the University of Leeds, in a paper on "The Reactivity of the Sulphur Linkage in Animal Fibres", to the oxidation of intact disulphide bonds or their hydrolysis products. More regulated changes of this nature occur in the milling, carrotting, crabbing and blowing processes. Dr. Speakman produced evidence to show that the cystine disulphide cross-linkage is more susceptible to hydrolysis in the stretched than it is in the unstretched fibre. He also showed that the contractile power of treated stretched fibres is dependent on the extent of the hydrolysis of the cystine disulphide cross-linkages, which vary with the $p \mathrm{H}$ value of the solution in which the fibre is treated. 
Raw wool may contain fifty per cent of its weight of grease, suint, dirt and vegetable matter. Its clean. ing is, therefore, an intricate process, and was the subject of two papers from the Wool Industries Research Association. Some of the fundamental principles of washing raw wool with solutions of soap and soda were dealt with by Dr. H. Phillips, who showed that the $p \mathrm{H}$ values of the detergent solutions used influence their wetting and emulsifying powers, and the stability of the emulsions which they form. Variations in the relative proportions of the different impurities in raw wool, caused by variations in the health of the sheep and the climatic conditions, have to be met by alterations in the washing process, which can only be foreseen when the process is under scientific control. Inherent weaknesses in the process are the effect of the alkali used on the wool and the incomplete removal of vegetable matter.

Dr. S. Townend described the frosted wool process, recently developed in the United States, by which it is elaimed that large quantities of vegetable matter and grease can be removed from raw wool without subjecting it to alkaline solutions. The wool is cooled to $-40^{\circ} \mathrm{F}$., and the frozen grease and vegetable matter are broken up and removed by mechanical treatment. The process removes more vegetable matter than the normal washing process, but the finer qualities of wool still require carbonising after treatment. The grease content of the wool cleaned by this process is also relatively high, and for certain purposes the wool has to be washed with soap and soda.

\section{Chemical Engineering Congress of the World Power Conference}

$\mathrm{H}$ R.H. THE DUKE OF KENT, in opening on Monday, June 22, the First International Chemical Engineering Congress at the Central Hall, Westminster, and welcoming delegates and members from thirty-seven countries, said: "Proud as each country is of the achievements of her great scientists and inventors, their work once done becomes international and contributes to the well-being and happiness of every race". The Right Hon. Viscount Leverhulme, in his presidential address which followed, dealt with the subject of chemical engineering and stated that the conception of holding an international congress of this kind was due to the late Sir Frederic Nathan, and that the development of that ideal into the present meeting was brought about by the World Power Conference.

About 120 papers from fifteen different countries were presented for discussion, whilst the membership numbered 850 , comprising about 350 representatives from thirty-six overseas countries.

Two technical sessions were held before lunch and two in the afternoon on Tuesday, June 23, and Friday, June 26, but as visits to colleges, research institutions and works in and around London had been arranged for Wednesday afternoon and Thursday afternoon, the technical sessions on these days were limited to the forenoon.

Since chemical plant may have to resist corrosive conditions and may have to withstand high tem. peratures and/or high pressures, both the material of which it is constructed and the method of fabrication are important factors to the chemical engineer. It was therefore not surprising that the papers and discussions at the first two sessions on Tuesday morning were devoted to materials of construction. At the first of these, eight papars were presented dealing with ferrous metals and their alloys designed to resist heat, rust and acid corrosion, the forging of autoclaves and vessels for withstanding liquids at high temperatures and pressures. Nor was cast iron omitted, for included in this section were papers showing the result of recent research work on this material and its application in chemical industry, as well as others on the prevention of corrosion in underground pipe lines made of ferrous metals. The following session covered a much wider field since it contained papers on refractory materials, stoneware, plastics, rubber and such lesser known fibres as jute, coir, and sisal, as well as non-ferrous metals and their alloys, and their application in the construction of chemical plant.

Separation in the chemical industry is such a wide subject that at the third session, after lunch, the papers ranged from the theory of coal-washing through the recovery of benzol and the removal of carbon monoxide from town's gas to the production and treatment of road tars and to problems connected with the distillation of absolute alcohol, fractionation of heavy oils, modern cracking pro. cesses and graphical calculations relating to plate columns. Included in this section were other papers on filtration, recent developments in evaporation, crystallization, solvent extraction and drying.

The fourth session, which followed immediately, contained several papers relating to some interesting aspects of size reduction, among which was one describing a plant wherein low-grade fuel such as lignite can be pulverized and fired into the furnace of a boiler in one process, the boiler operating at a very high efficiency.

Another group of papers in this session considered electro-metallurgical and electro-chemical industries, as well as the electrolytic treatment of water for the prevention of corrosion and boiler scale and methods of removing small quantities of iron from soluble alumin. ium salts, the pasteurization of liquids, anodic oxida. tion of aluminium and are welding of low carbon steels.

At the first session on Wednesday morning, the subject under discussion was destructive distillation, in which in addition to a paper on the underground gassification of coal, there was a number of papers on the problems connected with the manufacture of water gas and town's gas from lignite and similar fuels, as well as several papers on the various aspects of the development which has taken place in the gas in. dustry in various parts of the world.

With the rapid growth of large industrial units, the disposal of waste material, whether solid, liquid or gaseous, has become a pertinent and pressing problem to the chemical engineer. It formed the subject of several papers at the next session. Coupled with this series were other papers which showed the recent developments which have taken place in the preparation and treatment of lubricating oils. 